\title{
On hyperbolic Clifford algebras With INVOLUTION*
}

\author{
M. G. Mahmoudi
}

November 6, 2008

\begin{abstract}
The aim of this article is to provide a characterization of quadratic forms of low dimension such that the canonical involutions of the their Clifford algebras are hyperbolic.
\end{abstract}

\section{Introduction}

Clifford algebra is an important invariant associated to a quadratic form. Many properties of other invariants of quadratic forms like discriminant, Hasse invariant and Witt invariant can be better understood in the context of Clifford algebras. To every $n$-dimensional quadratic form $q$ over a field $K$, one can, in a canonical way, associate a $2^{n}$-dimensional $K$-algebra which is denoted by $C(q)$ or $C(V, q)$ where $V$ is the underlying vector space $q$. This $K$-algebra has an important subalgebra called the "even Clifford algebra" of $q$ which is denoted by $C_{0}(q)$ or $C_{0}(V, q)$. The algebra $C_{0}(q)$ is a $2^{n-1}$ dimensional $K$-algebra. If $n$ is even $C(q)$ is a $K$-central simple algebra and if $n$ is odd, $C_{0}(q)$ is a $K$-central simple algebra. In the Brauer group of $K$, the class of $C(q)$ if $n$ is even and the class of $C_{0}(q)$ if $n$ is odd coincides with the Witt invariant of $q$.

An involution $\varphi$ of a ring $R$ (with unity) is a bijective map $\varphi: R \rightarrow R$ which satisfies $\varphi(x+y)=\varphi(x)+\varphi(y), \varphi(x y)=\varphi(y) \varphi(x)$ and $\varphi(\varphi(x))=$ $x$ for all $x, y \in R$. Many algebras associated to a quadratic form are naturally endowed with involutions. A typical example is the endomorphism algebra $\operatorname{End}(V)$ (where $V$ is the underlying vector space of the quadratic form $q$ ). This algebra has a natural involution which is the adjoint map $\sigma_{q}$ : $\operatorname{End}(V) \rightarrow \operatorname{End}(V)$ satisfying $b_{q}(x, f(y))=b_{q}\left(\sigma_{q}(f) x, y\right)$ for every $x, y \in V$ and $f \in \operatorname{End}(V)$, here $b_{q}$ is the symmetric bilinear form associated to $q$, i.e., $b_{q}(x, y)=\frac{1}{2}(q(x+y)-q(x)-q(y))$. The map $\sigma_{q}$ is an involution of $\operatorname{End}(V)$ which also satisfies $\sigma_{q}(a f)=a \sigma_{q}(f)$, i.e., $\sigma_{q}$ is $K$-linear. Clifford

${ }^{*} 2000$ Mathematics Subject Classification: 11E81, 11E88 
algebra $C(V, q)$ also has natural involutions: the identity map id : $V \rightarrow V$ and - id $: V \rightarrow V$ induce involutions over $C(q)$ which are denoted by $J^{\text {id }}$ and $J^{- \text {id }}$ respectively. These involutions have the same restrictions to the even Clifford algebra, this restriction is denoted by $J$.

Many properties of a quadratic form $q$ can be conveniently expressed in terms of the algebra with involution $\left(\operatorname{End}(V), \sigma_{q}\right)$. Investigating the properties of an algebra with involution $(A, \sigma)$ which have counterparts in the algebraic theory of quadratic forms has been the subject of extensive study in the literature. One of these properties is hyperbolicity. Hyperbolic algebra with involutions can be intrinsically defined in such a way that the hyperbolicity of the algebra with involution $\left(\operatorname{End}(V), \sigma_{q}\right)$ is equivalent to the hyperbolicity of $q$ (c.f. [1] or [5, Ch. II]).

A natural question which arises is the following:

Question 1.1. Suppose that $\left(C(q), J^{\text {id }}\right)$ or $\left(C(q), J^{- \text {id }}\right)$ or $\left(C_{0}(q), J\right)$ are hyperbolic. What conclusion can one draw about $q$ ?

The aim of this article is study this question for quadratic forms of low dimension. The main results of this article can be summarized as follows:

Theorem 1.2. Let $(V, q)$ be a quadratic space with $\operatorname{dim} q \leqslant 8$. Then the algebra with involution $\left(C_{0}(V, q), J\right)$ is a hyperbolic if and only if $q$ contains a subform $q_{0}$ of trivial discriminant with $\operatorname{dim} q_{0} \equiv 2(\bmod 4)$.

Theorem 1.3. Let $(V, q)$ be a quadratic space with $\operatorname{dim} q \leqslant 5$. Then the algebra with involution $\left(C(V, q), J^{\mathrm{id}}\right)$ is a hyperbolic if and only if $q$ contains a subform $q_{0}$ of trivial discriminant with $\operatorname{dim} q_{0} \equiv 2$ or $3(\bmod 4)$.

Theorem 1.4. Let $(V, q)$ be a quadratic space with $\operatorname{dim} q \leqslant 5$. Then the algebra with involution $\left(C(V, q), J^{-\mathrm{id}}\right)$ is a hyperbolic if and only if $q$ contains a subform $q_{0}$ of trivial discriminant with $\operatorname{dim} q_{0} \equiv 1$ or $2(\bmod 4)$.

If $\operatorname{dim} q=6$ then $\left(C(V, q), J^{-\mathrm{id}}\right)$ is a hyperbolic if and only if $q \perp\left\langle-1, \mathrm{~d}_{ \pm} q\right\rangle$ is isotropic.

The proofs of these statements are given in $\S 3$ where for each dimension a more detailed statement is provided.

The Book of Involutions [5] and the articles [2], [3], [4] which have been sources of inspiration for our work, contain some special cases of these theorems, either implicitly or as a result of more general theorems. Our approach even for these known cases is different.

\section{Preliminaries}

All fields considered in this article are supposed to be of characteristic different from 2 . 


\subsection{Quadratic forms}

The reader is referred to the books $[7,9,10]$ for basic notions of quadratic forms. We just recall some notations for later use. If $(V, q)$ is a quadratic space over a field $K$ where $V$ is the underlying vector space of $q$, a diagonalization of $q$ with respect to an orthogonal basis $\left\{e_{1}, \cdots, e_{n}\right\}$ with $q\left(e_{1}\right)=a_{1}, q\left(e_{2}\right)=e_{2}, \cdots, q\left(e_{n}\right)=a_{n}$ is denoted by $\left\langle a_{1}, \cdots, a_{n}\right\rangle$. The discriminant (signed determinant) of $q$ which is denoted by $d_{ \pm}(q)$ is defined by $d_{ \pm}(q)=(-1)^{\frac{n(n-1)}{2}} a_{1} \cdots a_{n} \in K^{\times} / K^{\times 2}$.

\subsection{Hermitian forms and involutions}

Let $K$ be a field and let $A$ be a $K$-central simple algebra and let $\sigma$ be an involution of $A$. Let $V$ be a simple left $A$-module, by Schur lemma $D=\operatorname{End}_{A}(V)$ is division algebra. By Wedderburn-Artin theory $V$ has a right $D$-module structure and $A=\operatorname{End}_{D}(V)$. It is known that $\sigma$ is adjoint of some $\varepsilon$-hermitian form $h: V \times V \rightarrow D$ with respect to some involution $\tau$ on $D$, i.e., $h(x, f(y))=h(\sigma(f)(x), y)$ for all $x, y \in V$ and $f \in \operatorname{End}_{D}(V)$. We recall that an $\varepsilon$-hermitian form $h: V \times V \rightarrow D$ with respect to the involution $\tau$ (where $\varepsilon= \pm 1$ ) is bi-additive map which satisfies $h(x a, y b)=\tau(a) h(x, y) b$ and $h(x, y)=\varepsilon \tau(h(y, x))$ for all $x, y \in V$ and $a, b \in D$. If $\varepsilon=1$ (resp. $\varepsilon=-1$ ), an $\varepsilon$-hermitian form $(V, h)$ is also called a hermitian form (resp. skew hermitian form) with respect to the involution $\tau$.

If $(A, \sigma)$ is a $K$-central simple algebra with involution, the set of all symmetric elements of $K$ with respect to $\sigma$, i.e., elements $a \in K$ such that $\sigma(a)=a$, form a subfield $k$. It is known that either $K=k$ or $K / k$ is a quadratic extension. In the first case, $\sigma$ is said to be of the first kind, in the second case, $\sigma$ is said to be of the second kind. The sets $A^{+}=\{x \in A$ : $\sigma(x)=x\}$ (resp. $A^{-}=\{x \in A: \sigma(x)=-x\}$ ), i.e., the set of symmetric (resp. skew symmetric) elements of $A$ with respect to the involution $\sigma$ form a vector space over $k$. If $\operatorname{dim}_{k} A^{+}>\operatorname{dim}_{k} A^{-}$the involution $\sigma$ is said to be of orthogonal type. If $\operatorname{dim}_{k} A^{-}>\operatorname{dim}_{k} A^{+}$, the involution $\sigma$ is said to be of symplectic type. If $\operatorname{dim}_{k} A^{+}=\operatorname{dim}_{k} A^{-}$, the involution $\sigma$ is said to be of unitary type.

If $\sigma$ is of orthogonal type, Knus, Parimala and Sridharan [6] showed that the square class of the reduced norm of an invertible skew-symmetric element $a$ of $A$ is independent from the choice of $a$. As a result, the discriminant of $\sigma$ which is denoted by $\operatorname{disc}(\sigma)$, is defined in [5] as the square class of $(-1)^{m} \operatorname{Nrd}(a)$ where $m=\frac{1}{2} \operatorname{deg} A$.

\subsection{Hyperbolic and isotropic involutions}

An central simple algebra with involution $(A, \sigma)$ is said to be hyperbolic if $\sigma$ is adjoint of a hyperbolic $\varepsilon$-hermitian form over $D$ where $D$ is the 
division algebra defined in the previous paragraph. It is known (c.f. [1]) that $(A, \sigma)$ is hyperbolic if and only if $A$ contains an idempotent $e$ such that $\sigma(e)=1-e$. The last condition is also equivalent to the existence of a skew symmetric element $f \in A$, i.e., $\sigma(f)=-f$, such that $f^{2}=1$ (it suffices to put $f:=2 e-1)$.

It is necessary to extend this definition of hyperbolicity to algebras with involution of the form $\left(A_{0} \times A_{0}^{o p}, \sigma\right)$, where $A_{0}$ is a central simple algebra, and $A_{0}^{o p}$ is the opposite algebra and $\sigma$ is the exchange involution $\sigma\left(a, b^{o p}\right)=$ $\left(b, a^{o p}\right)$. This convention is specially useful in the context of Clifford algebras.

A central simple algebra with involution $(A, \sigma)$ is called isotropic if there exists an nonzero element $a \in A$ such that $\sigma(a) a=0$.

\subsection{Clifford algebras}

We use standard isomorphism theorems of Clifford algebras like $C\left(q^{\prime} \perp q\right) \simeq$ $C\left(q^{\prime}\right) \otimes C\left(\mathrm{~d}_{ \pm} q^{\prime} \cdot q\right)$ where $q^{\prime}$ is an even-dimensional form and $C_{0}\left(q^{\prime} \perp q\right) \simeq$ $C_{0}(q) \otimes C\left(-\mathrm{d}_{ \pm} q \cdot q\right)$ where $q^{\prime}$ is an odd dimensional form and $C_{0}(a \cdot q) \simeq C_{0}(q)$ where $a$ is a nonzero scalar: see [7]. See also [8] for an involutorial version.

\section{Hyperbolic Clifford algebras}

\subsection{General sufficient conditions}

We have the following result from [5, Proposition 8.5] and [2, Lemma 1.1]:

Proposition 3.1. The involutions $J^{\mathrm{id}}, J^{-\mathrm{id}}$ and $J$ are hyperbolic if $(V, q)$ is isotropic.

For the convenience of the reader we give the argument here: first note that it suffices to show the hyperbolicity of $J$; the hyperbolicity of $J^{\text {id }}$ and $J^{- \text {id }}$ then follows. As $q$ is isotropic there exist two vectors $x, y \in V$ such that $q(x)=q(y)=0$ and $b_{q}(x, y)=\frac{1}{2}$. Now consider the element $e=x \cdot y \in$ $C_{0}(V, q)$. We have $e^{2}=e$ and $J(e)=1-e$, hence $J$ is hyperbolic.

In the next proposition we provide less stringent sufficient conditions which imply the hyperbolicity of $J^{\text {id }}, J^{- \text {id }}$ or $J$ :

Proposition 3.2. Let $(V, q)$ be a quadratic space over a field $K$.

(i) If $q$ contains a subform $q_{0}$ with trivial discriminant and $\operatorname{dim} q_{0} \equiv$ 2 or $3(\bmod 4)$ then $J^{\mathrm{id}}$ is hyperbolic.

(ii) If $q$ contains a subform $q_{0}$ with $\mathrm{d}_{ \pm} q_{0}=1$ and $\operatorname{dim} q_{0} \equiv 1$ or $2(\bmod 4)$ then $J^{- \text {id }}$ is hyperbolic.

(iii) If $q$ contains a subform $q_{0}$ with $\mathrm{d}_{ \pm} q_{0}=1$ and $\operatorname{dim} q_{0} \equiv 2(\bmod 4)$ then $J$ is hyperbolic. 
Proof. Let $\left\{f_{1}, f_{2} \cdots, f_{m}\right\} \subset V$ be an orthogonal basis of $q_{0}$. We consider the product $f=f_{1} \cdot f_{2} \cdots f_{m} \in C(V, q)$.

If $m \equiv 2$ or $3(\bmod 4)$, we have $J^{\text {id }}(f)=-f$. We also have $f^{2}=\mathrm{d}_{ \pm} q_{0}=$ 1 , so $J^{\text {id }}$ is hyperbolic in this case.

If $m \equiv 1$ or $2(\bmod 4)$, we have $J^{-\mathrm{id}}(f)=-f$. We also have $f^{2}=$ $\mathrm{d}_{ \pm} q_{0}=1$, so $J^{- \text {id }}$ is hyperbolic.

If $m \equiv 2(\bmod 4)$, we have $f \in C_{0}(V, q)$. As $J(f)=-f$ and $f^{2}=1$ we conclude that $J$ is hyperbolic.

\subsection{Special necessary and sufficient conditions}

The Clifford algebra of a one dimensional form $q$ over a field $K$ is isomorphic to $K\left(\sqrt{\mathrm{d}_{ \pm} q}\right)$ if $\mathrm{d}_{ \pm} q \neq 1$ and is isomorphic to $K \times K$ if $\mathrm{d}_{ \pm} q=1$. In the first case the involution $J^{- \text {id }}$ is the nontrivial $K$-automorphism of $K\left(\sqrt{\mathrm{d}_{ \pm} q}\right)$ and in the later case $J^{- \text {id }}$ is the exchange involution of $K \times K$. The even Clifford algebra of $q$ is $K$ and $J$ is identity map of it. These well-known informations are summarized as the following:

Proposition 3.3. Let $(V, q)$ be a one dimensional quadratic space over a field $K$.

(i) The involution $J^{\text {id }}$ is identity map hence it is not hyperbolic.

(ii) The involution $J^{- \text {id }}$ is hyperbolic if and only if $d_{ \pm} q=1$.

(iii) The involution $J$ is the identity map hence it is not hyperbolic.

The characterization of the quadratic form $q$ with $\operatorname{dim} q=2$ for which the associated canonical involutions of Clifford algebra are hyperbolic which is given in Corollary 3.5 can be obtained using the following known result:

Proposition 3.4. Let $(Q, \sigma)$ be a quaternion algebra with involution over a field $K$.

(i) If $\sigma$ is orthogonal then $\sigma$ is hyperbolic if and only if $\operatorname{disc} \sigma=1$.

(ii) If $\sigma$ is symplectic then $\sigma$ is hyperbolic if and only if $Q$ splits.

Proof. For (i) see [3, Proposition 2.1]. For (ii) see [4, Lemma 16].

Corollary 3.5. Let $(V, q)$ be a two dimensional quadratic space over a field $K$.

(i) The involution $J^{\text {id }}$ is hyperbolic if and only if $q$ is isotropic.

(ii) The involution $J^{- \text {id }}$ is hyperbolic if and only if $q$ represents 1. 
(iii) The involution $J$ is hyperbolic if and only if $q$ is isotropic.

Proof. The involution $J^{\text {id }}$ is orthogonal. By Proposition 3.4 we deduce that it is hyperbolic if and only if $\operatorname{disc}\left(J^{\text {id }}\right)=1$. Let $q \simeq\langle a, b\rangle$ a diagonalization of $q$ and let $e_{1}, e_{2}$ be two orthogonal vector of $V$ with respect to $q$, such that $q\left(e_{1}\right)=a, q\left(e_{2}\right)=b$. We consider the element $f=e_{1} \cdot e_{2} \in C(V, q)$. We have $f^{2}=-a b$ and $J^{\text {id }}(f)=-f$. As $f$ is an skew symmetric element of $C(V, q)$ with respect to $J^{\text {id }}$, the square class of its reduced norm coincides with the discriminant of $J^{\text {id }}$. The reduced norm of $f$ is $-a b$ thus $\mathrm{d}_{ \pm} q=1$. We conclude that $q$ is isotropic. This proves $(i)$.

As the involution $J^{- \text {id }}$ is symplectic, by previous proposition, it is hyperbolic if and only if $C(V, q)$ splits. Now keeping the notations of the previous paragraph, $C(V, q)$ is isomorphic as $K$-algebra with the quaternion algebra $(a, b)_{K}$ generated by $i=e_{1}, j=e_{2}$ subject to the relations: $i^{2}=a$, $j^{2}=b$ and $i j=-j i$. It is known that this algebra splits if and only if the four dimensional form $\langle 1,-a,-b, a b\rangle$ is hyperbolic. This implies that $q \simeq\langle a, b\rangle \simeq\langle 1, a b\rangle$. Thus $q$ represents 1 and the proof of $(i i)$ is done.

To prove (iii) note that when $\operatorname{dim} V=2$, the hyperbolicity of $J^{\text {id }}$ and $J$ are equivalent.

Proposition 3.6. Let $(V, q)$ be a three dimensional quadratic space over a field $K$.

(i) $J^{\text {id }}$ is hyperbolic if and only if $q$ is isotropic or $\mathrm{d}_{ \pm} q=1$.

(ii) $J^{- \text {id }}$ is hyperbolic if and only if $q$ represents 1.

(iii) $J$ is hyperbolic if and only if $q$ is isotropic.

Proof. Let $q \simeq\langle a, b, c\rangle$ a diagonalization of $q$ and let $\left\{e_{1}, e_{2}, e_{3}\right\}$ be an orthogonal basis of $V$ with respect to $q$, such that $q\left(e_{1}\right)=a, q\left(e_{2}\right)=b$ and $q\left(e_{3}\right)=c$.

(i) As $J^{\text {id }}$ is hyperbolic, there exists an skew symmetric element $z \in$ $C(V, q)$ with respect to $J^{\text {id }}$ such that $z^{2}=1$. We can write $z=\lambda_{12} e_{1} e_{2}+$ $\lambda_{13} e_{1} e_{3}+\lambda_{23} e_{2} e_{3}+\lambda_{123} e_{1} e_{2} e_{3}$ where $\lambda_{12}, \lambda_{13}, \lambda_{23}, \lambda_{123} \in K$. From the relation $z^{2}=1$ we obtain: $\lambda_{12} \lambda_{123}=\lambda_{13} \lambda_{123}=\lambda_{23} \lambda_{123}=0$ and $-a b \lambda_{12}^{2}-$ $a c \lambda_{13}^{2}-b c \lambda_{23}^{2}-a b c \lambda_{123}^{2}=1$. If $\lambda_{123}=0$, the form $q^{\prime}=\langle-a b,-a c,-b c\rangle$ represents 1 . As $\mathrm{d}_{ \pm} q^{\prime}=1$, we deduce that $q^{\prime}$ is isotropic so $q$ is isotropic as well. If $\lambda_{123} \neq 0$, we obtain $\lambda_{12}=\lambda_{13}=\lambda_{23}=0$. We obtain $-a b c \in K^{\times^{2}}$ so $\mathrm{d}_{ \pm} q=1$.

(ii) As $J^{-d}$ is hyperbolic, there exists an skew symmetric element $z \in$ $C(V, q)$ with respect to $J^{- \text {id }}$ such that $z^{2}=1$. We can write $z=\lambda_{1} e_{1}+$ $\lambda_{2} e_{2}+\lambda_{3} e_{3}+\lambda_{12} e_{1} e_{2}+\lambda_{13} e_{1} e_{3}+\lambda_{23} e_{2} e_{3}$ where $\lambda_{1}, \lambda_{2}, \lambda_{3}, \lambda_{12}, \lambda_{13}, \lambda_{23} \in K$. From the relation $z^{2}=1$ we obtain $z^{2}=\lambda_{1}^{2} a+\lambda_{2}^{2} b+\lambda_{3}^{2} c-\lambda_{12}^{2} a b-\lambda_{13}^{2} a c-$ $\lambda_{23}^{2} b c+2\left(\lambda_{1} \lambda_{23}-\lambda_{2} \lambda_{13}+\lambda_{3} \lambda_{12}\right) e_{1} e_{2} e_{3}=1$. We then have $\lambda_{1}^{2} a+\lambda_{2}^{2} b+\lambda_{3}^{2} c-$ 
$\lambda_{12}^{2} a b-\lambda_{13}^{2} a c-\lambda_{23}^{2} b c=1$ and $\lambda_{1} \lambda_{23}-\lambda_{2} \lambda_{13}+\lambda_{3} \lambda_{12}=0$. We have two cases: $-a b c \in K^{\times 2}$ and $-a b c \notin K^{\times 2}$. In the first case let $d \in K$ an element such that $d^{2}=-a b c$. We obtain

$$
\begin{aligned}
1= & \lambda_{1}^{2} a+\lambda_{2}^{2} b+\lambda_{3}^{2} c-\lambda_{12}^{2} a b-\lambda_{13}^{2} a c-\lambda_{23}^{2} b c \\
= & \lambda_{1}^{2} a+\lambda_{2}^{2} b+\lambda_{3}^{2} c-\lambda_{12}^{2} a b-\lambda_{13}^{2} a c-\lambda_{23}^{2} b c+ \\
& 2 d\left(\lambda_{1} \lambda_{23}-\lambda_{2} \lambda_{13}+\lambda_{3} \lambda_{12}\right) \\
= & a\left(\lambda_{1}+a^{-1} d \lambda_{23}\right)^{2}+b\left(\lambda_{2}-b^{-1} d \lambda_{13}\right)^{2}+c\left(\lambda_{3}+c^{-1} d \lambda_{12}\right)^{2}
\end{aligned}
$$

So in this case, $q$ represents 1 . Now suppose that $-a b c \notin K^{\times 2}$. We have an isomorphism of algebras with involution

$$
\left(C(\langle a, b, c\rangle), J^{-\mathrm{id}}\right) \simeq\left(C(\langle a, b\rangle), J^{-\mathrm{id}}\right) \otimes\left(C(\langle-a b c\rangle), J^{\mathrm{id}}\right) .
$$

Note that $C(\langle-a b c\rangle) \simeq K(\sqrt{-a b c})$ is a quadratic extension of $K$ and $J^{\text {id }}$ restricts the identity map of this extension. Using Proposition 3.4 (ii) we deduce that the quaternion algebra $C(\langle a, b\rangle) \simeq(a, b)_{K}$ splits over $K(\sqrt{-a b c})$. This shows that the forms $\langle 1,-a,-b, a b\rangle$ which is the norm form of $q$ is hyperbolic over $K(\sqrt{-a b c})$. It follows that $\langle a, b\rangle$ represents 1 over $K(\sqrt{-a b c})$. This readily implies that $\langle a, b, c\rangle$ represents 1 over $K$ which concludes the proof.

(iii) In this case $\lambda_{123}$ is necessarily 0 so as in the first part of the we deduce that $q$ is isotropic.

Notation 3.7. Let $(A, J)$ be a finite dimensional algebra with involution over a field $K$. The set of symmetric elements of $A$ with respect to $J$ with trace zero is denoted by $\operatorname{sym}^{0}(A, J)$ or simply by $\operatorname{sym}^{0}(J)$.

Proposition 3.8. Let $(V, q)$ be a five dimensional quadratic space over a field $K$. Then $J$ is hyperbolic if and only if $q$ is isotropic. (See [5, Prop. 15.21])

Proof. Let $q \simeq\langle a, b, c, d, e\rangle$ be a diagonalization of $q$ and let $\left\{e_{1}, e_{2}, e_{3}, e_{4}, e_{5}\right\}$ be an orthogonal basis of $V$ with respect to $q$ such that $q\left(e_{1}\right)=a, q\left(e_{2}\right)=b$, $q\left(e_{3}\right)=c, q\left(e_{4}\right)=d$ and $q\left(e_{5}\right)=e$.

For every $x \in \operatorname{sym}^{0}(J)$ we have $x^{2} \in K$. Consider the map $s: \operatorname{sym}^{0}(J) \rightarrow$ $K$ defined by $s(x)=x^{2}$. We have an isomorphism $(V, q) \simeq\left(\operatorname{sym}^{0}(J), s\right)$ defined by $x \mapsto e_{1} e_{2} e_{3} e_{4} e_{5} x$. So it is enough to show that $\left(\operatorname{sym}^{0}(J), s\right)$ is isotropic. The involution $J$ is symplectic. As $J$ is hyperbolic, there is a decomposition $\left(C_{0}(V, q), J\right) \simeq\left(M_{2}(K), \rho\right) \otimes(Q, \tau)$, where $\rho$ is the canonical involution of $M_{2}(K)$ and $\tau$ is an orthogonal involution of a quaternion algebra $Q$ over $K$ (see [1, Theorem 2.2]). Let $y \in Q$ a skew symmetric element with $y^{2}=\alpha \in K^{\times}$. We consider two symmetric elements $s_{1}=\left(\begin{array}{cc}0 & 1 \\ -1 & 0\end{array}\right) \otimes y$ 
and $s_{2}=\left(\begin{array}{cc}1 & 0 \\ 0 & -1\end{array}\right) \otimes y$ in $\operatorname{sym}^{0}(J)$. We have $s_{1}^{2}=-\alpha$ and $s_{2}^{2}=\alpha$. We conclude that $\left(\operatorname{sym}^{0}(J), s\right)$ is isotropic.

Proposition 3.9. Let $(V, q)$ be a four dimensional quadratic space over a field $K$.

(i) $J$ is hyperbolic if and only if $q$ is isotropic. (See [5, Prop. 15.14])

(ii) $J^{- \text {id }}$ is hyperbolic if and only if $q$ represents 1.

(iii) $J^{\text {id }}$ is hyperbolic if and only if $q$ represents $-\mathrm{d}_{ \pm} q$.

Proof. Let $q \simeq\langle a, b, c, d\rangle$ a diagonalization of $q$ and let $\left\{e_{1}, e_{2}, e_{3}, e_{4}\right\}$ be an orthogonal basis of $V$ with respect to $q$ such that $q\left(e_{1}\right)=a, q\left(e_{2}\right)=b$, $q\left(e_{3}\right)=c$ and $q\left(e_{4}\right)=d$.

(i) First we suppose that $\mathrm{d}_{ \pm} q=1$. As scaling $q$ does not, up to isomorphism, affect $\left(C_{0}(q), J\right)$, we may assume that $a=1$ and $d=b c$ so we have $q \simeq\langle 1, b, c, b c\rangle$ and $\left(e_{1} e_{2}\right)^{2}=-b$ and $\left(e_{1} e_{3}\right)^{2}=-c$. Let $Q$ be the quaternion algebra generated by $i=e_{1} e_{2}, j=e_{1} e_{3}$ over $K$. We have $Q \simeq(-b,-c)_{K}$. We can write $C_{0}(V, q)=Q \oplus Q \cdot\left(e_{1} e_{2} e_{3} e_{4}\right)$. We have the isomorphism $C_{0}(V, q) \simeq Q \times Q$ with $\left(x+y \cdot\left(e_{1} e_{2} e_{3} e_{4}\right)\right) \mapsto(x+y, x-y)$ for $x, y \in Q$. The involution $J$ of $C_{0}(V, q)$ corresponds to the involution $J^{*}: Q \times Q \rightarrow Q \times Q$ with $J^{*}(x, y)=(\gamma(x), \gamma(y))$ where $\gamma=\left.J\right|_{Q}$. Note that $\gamma$ is the canonical involution of $Q$. As $J$ is hyperbolic, there exists $(x, y) \in Q \times Q$ such that $(x, y)^{2}=1$ and $J^{*}(x, y)=-(x, y)$. This implies that $\gamma$ is hyperbolic and by Proposition 3.4, $Q$ is not a division algebra and $\langle 1, b, c, b c\rangle$ is isotropic.

Now suppose that $\delta=\mathrm{d}_{ \pm} q \neq 1$. In this case $C_{0}(V, q)$ is a quaternion algebra over $K(\sqrt{\delta})$ and $J$ is an involution of the first kind and of symplectic type. In particular $J$ is hyperbolic if and only if $C_{0}(V, q)$ splits. As $q \simeq$ $\langle a, b, c, d\rangle$ we have the isomorphism $C_{0}(V, q) \simeq(-a b,-a c)_{K(\delta)}$. The algebra $C_{0}(V, q)$ splits if and only if $\langle 1, a b, a c, b c\rangle$ is isotropic over $K(\sqrt{\delta})$. This readily implies that $q$ is isotropic.

An alternative proof may also be given using Proposition 3.6. We have the isomorphism of algebras with involution

$$
\left(C_{0}(q), J\right) \simeq\left(C(-d \cdot\langle a, b, c\rangle), J^{-\mathrm{id}}\right) .
$$

If $J$ is hyperbolic, using Proposition 3.6 we deduce that the three dimensional form $-d \cdot\langle a, b, c\rangle$ represents 1 over $K$. It follows that $\langle a, b, c\rangle$ represents $-d$ over $K$, hence $q \simeq\langle a, b, c, d\rangle$ is isotropic.

(ii) We have an isomorphism of algebras with involution $\left(C(q), J^{- \text {id }}\right) \simeq$ $\left(C_{0}(q \perp\langle-1\rangle), J\right)$. From Proposition 3.8 we deduce that $q \perp\langle-1\rangle$ is isotropic, therefore $q$ represents 1 .

There is however a more elementary proof in the case where $\mathrm{d}_{ \pm} q=1$. As $J^{- \text {id }}$ is hyperbolic, there exits a skew symmetric element $z \in C(V, q)$ 
with respect to $J^{- \text {id }}$ such that $z^{2}=1$. The element $z$ can be written as $z=\lambda_{1} e_{1}+\lambda_{2} e_{2}+\lambda_{3} e_{3}+\lambda_{4} e_{4}+\lambda_{12} e_{1} e_{2}+\lambda_{13} e_{1} e_{3}+\lambda_{14} e_{1} e_{4}+\lambda_{23} e_{2} e_{3}+$ $\lambda_{24} e_{2} e_{4}+\lambda_{34} e_{3} e_{4}$. We obtain $z^{2}=A+B+C+D$ where

$$
\begin{aligned}
\mathrm{A}= & \lambda_{1}^{2} a+\lambda_{2}^{2} b+\lambda_{3}^{2} c+\lambda_{4}^{2} d, \\
\mathrm{~B}= & -\lambda_{12}^{2} a b-\lambda_{13}^{2} a c-\lambda_{14}^{2} a d-\lambda_{23}^{2} b c-\lambda_{24}^{2} b d-\lambda_{34}^{2} c d, \\
\mathrm{C}= & \left(2 \lambda_{1} \lambda_{23}-2 \lambda_{2} \lambda_{13}+2 \lambda_{3} \lambda_{12}\right) e_{1} e_{2} e_{3}+ \\
& \left(2 \lambda_{1} \lambda_{24}-2 \lambda_{2} \lambda_{14}+2 \lambda_{4} \lambda_{12}\right) e_{1} e_{3} e_{4}+ \\
& \left(2 \lambda_{2} \lambda_{34}-2 \lambda_{3} \lambda_{24}+2 \lambda_{4} \lambda_{23}\right) e_{2} e_{3} e_{4} \\
\mathrm{D}= & \left(2 \lambda_{12} \lambda_{34}-2 \lambda_{13} \lambda_{24}+2 \lambda_{14} \lambda_{23}\right) e_{1} e_{2} e_{3} e_{4} .
\end{aligned}
$$

As $z^{2}=1 \in K$ so $C=D=0$ and we obtain $1=A+B$. As $\mathrm{d}_{ \pm} q=1$, there exists an element $\delta \in K$ such that $\delta^{2}=$ abcd. We then obtain $B=-a b\left(\lambda_{12}^{2}+\frac{\delta}{a b} \lambda_{34}\right)^{2}-a c\left(\lambda_{13}-\frac{\delta}{a c} \lambda_{24}\right)^{2}-a d\left(\lambda_{14}+\frac{\delta}{a d} \lambda_{23}\right)^{2}$. Now from the relation $A+B=1$ we deduce that the seven dimensional form $\langle a, b, c, d,-a b,-a c,-a d\rangle$ represents 1 . The eight dimensional form $q^{\prime}=$ $\langle 1,-a,-b,-c,-d, a b, a c, a d\rangle$ is so isotropic. We have

$$
q^{\prime} \simeq\langle 1,-a,-b,-c,-a b c, a b, a c, b c\rangle,
$$

so $q^{\prime}$ is a Pfister form, therefore $q^{\prime}$ is hyperbolic. We deduce that the form $\langle 1,-a,-b,-c,-d\rangle$ which is a Pfister neighbor of $q^{\prime}$ is isotropic. We conclude that $q$ represents 1 .

(iii) We have an isomorphism of algebras with involution

$$
\left.\left(C(\langle a, b, c, d\rangle), J^{\mathrm{id}}\right) \simeq C(\langle-c d a,-c d,-a b c,-a b d\rangle), J^{-\mathrm{id}}\right) .
$$

From (ii) we deduce that the four dimensional form $\langle-c d a,-c d,-a b c,-a b d\rangle$ represents 1. It follows that $q=\langle a, b, c, d\rangle$ represents $-a b c d=-\mathrm{d}_{ \pm} q$.

An alternative proof for the case where $\mathrm{d}_{ \pm} q=1$ can be given as the following. As $J^{\text {id }}$ is hyperbolic, there exits a skew symmetric element $z \in$ $C(V, q)$ with respect to $J^{\text {id }}$ such that $z^{2}=1$. We can write

$$
\begin{aligned}
z= & \left(\lambda_{12} e_{1} e_{2}+\lambda_{13} e_{1} e_{3}+\lambda_{14} e_{1} e_{4}+\lambda_{23} e_{2} e_{3}+\lambda_{24} e_{2} e_{4}+\lambda_{34} e_{3} e_{4}\right)+ \\
& \left(\lambda_{234} e_{2} e_{3} e_{4}+\lambda_{134} e_{1} e_{3} e_{4}+\lambda_{124} e_{1} e_{2} e_{4}+\lambda_{123} e_{1} e_{2} e_{3}\right)
\end{aligned}
$$

We obtain $z^{2}=A+B+C+D$ where

$$
\begin{aligned}
A= & -\lambda_{12}^{2} a b-\lambda_{13}^{2} a c-\lambda_{14}^{2} a d-\lambda_{23}^{2} b c-\lambda_{24}^{2} b d-\lambda_{34}^{2} c d, \\
B= & -\lambda_{234}^{2} b c d-\lambda_{134}^{2} a c d-\lambda_{124}^{2} a b d-\lambda_{123}^{2} a b c \\
C= & -2\left(\lambda_{23} \lambda_{123} b c+\lambda_{24} \lambda_{124} b d+\lambda_{34} \lambda_{134} c d\right) e_{1} \\
& +2\left(\lambda_{13} \lambda_{123} a c+\lambda_{14} \lambda_{124} a d+\lambda_{34} \lambda_{234} c d\right) e_{2} \\
& +2\left(-\lambda_{12} \lambda_{123} a b+\lambda_{14} \lambda_{134} a d+\lambda_{24} \lambda_{234} b d\right) e_{3} \\
& -2\left(\lambda_{12} \lambda_{124} a b+\lambda_{13} \lambda_{134} a c+\lambda_{23} \lambda_{234} b c\right) e_{4} \\
D= & 2\left(\lambda_{12} \lambda_{34}-\lambda_{13} \lambda_{24}+\lambda_{14} \lambda_{23}\right) e_{1} e_{2} e_{3} e_{4} .
\end{aligned}
$$


As $z^{2}=1 \in K$ so $C=D=0$. We obtain $z^{2}=A+B=1$. Consider an element $\delta \in K$ such that $\delta^{2}=a b c d$. Note that $-a b c d A=c d\left(\lambda_{12} a b+\right.$ $\left.\lambda_{34} \delta\right)^{2}+b d\left(\lambda_{13} a c-\lambda_{24} \delta\right)^{2}+b c\left(\lambda_{14} a d+\lambda_{23} \delta\right)^{2}$ and $-a b c d B=\left(\lambda_{124} b c d\right)^{2} a+$ $\left(\lambda_{134} a c d\right)^{2} b+\left(\lambda_{124} a b d\right)^{2} c+\left(\lambda_{123} a b c\right)^{2} d$. From the relation $-a b c d(A+B)=$ $-a b c d$ we deduce that the seven dimensional form $\langle c d, b d, b c, a, b, c, d\rangle$ represents $-a b c d$. So the eight dimensional form $q^{\prime}=\langle c d, b d, b c, a, b, c, d, a b c d\rangle$ is isotropic. We have $\langle c d, b d, b c, a, b, c, d, a b c d\rangle \simeq\langle c d, b d, b c, b c d, b, c, d, 1\rangle$ so $q^{\prime}$ is a Pfister form, consequently $q^{\prime}$ becomes hyperbolic. As $q^{\prime \prime}=\langle a, b, c, d, 1\rangle$ is a Pfister neighbor of $q^{\prime}$ so $q^{\prime \prime}$ is hyperbolic and we conclude that $q$ represents $-1$.

Proposition 3.10. Let $(V, q)$ be a five dimensional quadratic space over a field $K$. Then $J^{\text {id }}$ is hyperbolic if and only if $q$ contains a three dimensional subform of trivial discriminant.

Proof. Let $q \simeq\langle a, b, c, d, e\rangle$ be a diagonalization of $q$ and let $\left\{e_{1}, e_{2}, e_{3}, e_{4}, e_{5}\right\}$ be an orthogonal basis of $V$ with respect to $q$ such that $q\left(e_{1}\right)=a, q\left(e_{2}\right)=b$, $q\left(e_{3}\right)=c, q\left(e_{4}\right)=d$ and $q\left(e_{5}\right)=e$.

We have an isomorphism of algebras with involution

$$
\left(C(q), J^{\text {id }}\right) \simeq\left(C\left(q^{\prime}\right), J^{\text {id }}\right) \otimes(K(\sqrt{\delta}), \mathrm{id})
$$

where $q^{\prime}=\langle a, b, c, d\rangle$ and $\delta=\mathrm{d}_{ \pm} q=a b c d e$. From Proposition 3.9 (iii) we deduce that $q$ represents $-\mathrm{d}_{ \pm} q^{\prime}=-a b c d$ over $K(\sqrt{\delta})$. It follows that the form $\langle a, b, c, d, a b c d\rangle$ is isotropic over $K(\sqrt{\delta})$. Note that $\langle a, b, c, d, a b c d\rangle \simeq_{K(\sqrt{\delta})}$ $\langle a, b, c, d, e\rangle$. Therefore $q$ is isotropic over $K(\sqrt{\delta})$. This implies that $q$ a contains two dimensional form $\varphi$ similar to $\langle 1,-\delta\rangle$. The Witt complement of $\varphi$ in $q$ is the desired form.

Proposition 3.11. Let $(V, q)$ be a six dimensional quadratic space over a field $K$. Then $J$ is hyperbolic if and only if $\mathrm{d}_{ \pm} q=1$ or $q$ is isotropic. (See [5, Prop. 15.39])

Proof. Let $q \simeq\langle a, b, c, d, e, f\rangle$ be a diagonalization of $q$ and let $\left\{e_{1}, e_{2}\right.$, $\left.e_{3}, e_{4}, e_{5}, e_{6}\right\}$ be an orthogonal basis of $V$ with respect to $q$ such that $q\left(e_{1}\right)=$ $a, q\left(e_{2}\right)=b, q\left(e_{3}\right)=c, q\left(e_{4}\right)=d, q\left(e_{5}\right)=e$ and $q\left(e_{6}\right)=f$.

If $\mathrm{d}_{ \pm} q=1$ we have done. Suppose that $\delta=\mathrm{d}_{ \pm} q \neq 1$. In this case $C_{0}(V, q)$ is a biquaternion algebra over $K(\sqrt{\delta})$. We will show that if $J$ is hyperbolic then $q$ is isotropic. As for every $\alpha \in K^{\times}$we have an isomorphism of algebras with involution $\left(C_{0}(V, \alpha \cdot q), J\right) \simeq\left(C_{0}(V, q), J\right)$, by scaling we may assume that $f=1$ and so $q \simeq q_{0} \perp\langle 1\rangle$ where $q_{0}=\langle a, b, c, d, e\rangle$. We have $\mathrm{d}_{ \pm} q=-a b c d e$. Let $V_{0}$ be the underlying vector space of $q_{0}$. We have

$$
\left(C_{0}(V, q), J\right) \simeq\left(C_{0}\left(V_{0}, q_{0}\right) \otimes K\left(\sqrt{\mathrm{d}_{ \pm} q}\right), J \otimes \tau\right)
$$


where $\tau$ is the nontrivial automorphism of $K\left(\sqrt{\mathrm{d}_{ \pm} q}\right) / K$. Consider the element $\xi=e_{1} e_{2} e_{3} e_{4} e_{5} \in C_{0}\left(V_{0}, q_{0}\right)$. We have $\xi^{2}=a b c d e$. Consider the map $\varphi: V_{0} \rightarrow \operatorname{sym}^{0}\left(C_{0}\left(V_{0}, q_{0}\right), J\right)$ defined by $\varphi(v)=\xi v$. Let $s$ : $\operatorname{sym}^{0}\left(C_{0}\left(V_{0}, q_{0}\right), J\right) \rightarrow K$ defined $s(x)=x^{2}$. The map $\varphi$ induces an isomorphism of quadratic spaces $\left(V_{0}, q_{0}\right) \simeq\left(\operatorname{sym}^{0}\left(C_{0}\left(V_{0}, q_{0}\right), J\right), s\right)$. It is enough to show that $q_{0}$ represents -1 . As $\left(C_{0}(V, q), J\right)$ is hyperbolic, there exists $z \in C_{0}\left(V_{0}, q_{0},\right)$ such that $J(z)=z$ and $z^{2}=\mathrm{d}_{ \pm} q$. Note that these relations imply that $z \in \operatorname{sym}^{0}\left(C_{0}\left(V_{0}, q_{0}\right), J\right)$. Let $w \in V_{0}$ such that $\varphi(w)=z$. We obtain then $\xi w=z$ and so $\xi^{2} w^{2}=z^{2}$ and this implies that $q_{0}(w)=w^{2}=$ $-1$.

Proposition 3.12. Let $(V, q)$ be a five dimensional quadratic space over a field $K$. Then $J^{-\mathrm{id}}$ is hyperbolic if and only if $q$ represents 1 or $\mathrm{d}_{ \pm} q=1$.

Proof. Let $q \simeq\langle a, b, c, d, e\rangle$ be a diagonalization of $q$ and let $\left\{e_{1}, e_{2}, e_{3}, e_{4}, e_{5}\right\}$ be an orthogonal basis of $V$ with respect to $q$ such that $q\left(e_{1}\right)=a, q\left(e_{2}\right)=b$, $q\left(e_{3}\right)=c, q\left(e_{4}\right)=d$ and $q\left(e_{5}\right)=e$.

We have an isomorphism of algebras with involution $\left(C(q), J^{- \text {id }}\right) \simeq$ $\left(C_{0}(q \perp\langle-1\rangle), J\right)$. Using Proposition 3.11 we deduce that $q \perp\langle-1\rangle$ is either isotropic or has trivial discriminant. This implies that either $q$ represents 1 or $\mathrm{d}_{ \pm} q=1$.

Proposition 3.13. Let $(V, q)$ be an eight dimensional quadratic space over a field $K$. Then $J$ is hyperbolic if and only if $q$ is isotropic or it contains a subform isometric to an Albert form.

Proof. First suppose that $\mathrm{d}_{ \pm} q=1$. We have an isomorphism $\left(C_{0}(q), J\right) \simeq$ $(A, \sigma) \times(A, \sigma)$ where $A$ is a central simple algebra of degree 8 with an involution $\sigma$ of trivial discriminant. By Triality Lemma (c.f. [5, 42.3] ) we have $\left(C_{0}(A, \sigma), \underline{\sigma}\right) \simeq\left(\right.$ End $\left.V, a d_{q}\right) \times(A, \sigma)$. By [2, 2.3], we deduce that $q \simeq \mathbb{H} \perp \alpha$ where $\alpha$ is an Albert form over $K$ and $\mathbb{H}$ is a hyperbolic plane over $K$.

Now suppose that $\delta=\mathrm{d}_{ \pm} q \neq 1$. If $q$ is isotropic we have done. We may then assume that $q$ is anisotropic. According to the previous paragraph, $\left.q\right|_{K(\sqrt{\delta})}$ is isotropic. We therefore obtain $q \simeq\langle\lambda,-\lambda \delta\rangle \perp \alpha$, where $\lambda \in K^{\times}$ and $\alpha$ is an Albert form over $K$.

Proposition 3.14. Let $(V, q)$ be a seven dimensional quadratic space over a field $K$. Then $J$ is hyperbolic if and only if $q$ contains an Albert form (or equivalently $J$ is hyperbolic if and only if $q$ represents its discriminant).

Proof. Let $\delta=\mathrm{d}_{ \pm} q$. Consider the form $q_{1}=q \perp\langle-\delta\rangle$. This form is an 8-dimensional form with trivial discriminant. The algebra $\left(C_{0}\left(q_{1}\right), J\right)$ is 
hyperbolic. According to $3.13, q_{1}$ is either isotropic or contains an Albert form. If $q_{1}$ is isotropic we deduce that $q \simeq\langle\delta\rangle \perp \alpha$ where $\alpha$ is an Albert form. If $q_{1}$ contains an Albert form then $q_{1}$ is isotropic as $\mathrm{d}_{ \pm} q_{1}=1$ and we are back to the first case.

Proposition 3.15. Let $(V, q)$ be a six dimensional quadratic space over a field $K$. Then $J^{- \text {id }}$ is hyperbolic if and only if $q \perp\left\langle-1, \mathrm{~d}_{ \pm} q\right\rangle$ is isotropic.

Proof. Let $q \simeq\langle a, b, c, d, e, f\rangle$ be a diagonalization of $q$ and let $\left\{e_{1}, e_{2}\right.$, $\left.e_{3}, e_{4}, e_{5}, e_{6}\right\}$ be an orthogonal basis of $V$ with respect to $q$ such that $q\left(e_{1}\right)=$ $a, q\left(e_{2}\right)=b, q\left(e_{3}\right)=c, q\left(e_{4}\right)=d, q\left(e_{5}\right)=e$ and $q\left(e_{6}\right)=f$.

We have an isomorphism of algebras with involution $\left(C(q), J^{- \text {id }}\right) \simeq$ $\left(C_{0}(q \perp\langle-1\rangle), J\right)$. As $\left(C(q), J^{- \text {id }}\right)$ is hyperbolic, so is $\left(C_{0}(q \perp\langle-1\rangle), J\right)$. From Proposition 3.14 we deduce that $\varphi=q \perp\langle-1\rangle$ contains an Albert form or equivalently $\varphi$ represents its discriminant. We have $\mathrm{d}_{ \pm} \varphi=-\mathrm{d}_{ \pm} q$. It follows that $q \perp\left\langle-1, \mathrm{~d}_{ \pm} q\right\rangle$ is isotropic.

Acknowledgment. I am indebted to Jean-Pierre Tignol for many inspiring discussions in Dublin (2005) and Oberwolfach (2006) which initiated this work, for helpful comments and suggesting to use the triality and Garibaldi's result for the proof of Proposition 3.13. I acknowledge the financial support provided by the Research Council of Sharif University of Technology.

\section{References}

[1] Bayer-Fluckiger, E.; Shapiro, D. B.; Tignol, J.-P.; Hyperbolic involutions. Math. Z. 214 (1993) 461-476.

[2] Garibaldi, R. Skip; Clifford algebras of hyperbolic involutions. Math. Z. 236 (2001), no. 2, 321-349.

[3] Haile, D. E.; Morandi, P. J.; Hyperbolicity of algebras with involution and connections with Clifford algebras. Comm. Algebra 29 (2001), no. $12,5733-5753$.

[4] Haile, D. E.; Tignol, J.-P.; Algebras with involution that become hyperbolic under a given extension. J. Algebra 199 (1998), no. 1, 94-115.

[5] Knus, Max-Albert; Merkurjev, Alexander; Rost, Markus; Tignol, JeanPierre; The book of involutions. American Mathematical Society Colloquium Publications, 44, American Mathematical Society, Providence, RI, 1998.

[6] Knus, M.-A.; Parimala, R.; Sridharan, R.; On the discriminant of an involution, Bull. Soc. Math. Belg. Sér. A 43 (1991), no. 1-2, 89-98. 
[7] Lam, T. Y.; Introduction to quadratic forms over fields. Graduate Studies in Mathematics 67. Providence, RI: American Mathematical Society (AMS). (2005)

[8] Lewis, D. W.; Periodicity of Clifford algebras and exact octagons of Witt groups. Math. Proc. Cambridge Philos. Soc. 98 (1985), no. 2, 263-269.

[9] Pfister, A.; Quadratic forms with applications to algebraic geometry and topology. London Mathematical Society Lecture Note Series. 217. Cambridge: Cambridge Univ. Press. (1995).

[10] Scharlau, W.; Quadratic and Hermitian forms. Grundlehren der Mathematischen Wissenschaften, 270. Springer-Verlag, Berlin, 1985.

Department of Mathematical Sciences, Sharif University of Technology, P.O. Box: 11155-9415, TEHRAN, IRAN. E-MAIL ADDRESS: mmahmoudi@sharif.ir 